\title{
Sciendo
}

\section{Memes and education: opportunities, approaches and perspectives}

Xie Dongqiang

Peoples' Friendship University of Russia, e-mail:1032195620@rudn.ru

Ludovico De Serio

University of Genoa

e-mail:ludovico.deserio@gmail.com

Alexander Malakhov,

Peoples' Friendship University of Russia

e-mail:1032193337@rudn.ru

Olga Matys

Peoples' Friendship University of Russia Eurostat database (Comext) and Eurostat estimates e-mail:1032193346@rudn.ru

DOI: $10.2478 /$ gssfj-2020-0009

\begin{abstract}
This article presents the results of an international research of using Internet-memes in education process. The writing team set out to explore the reasons and ways of using Internet memes in education in different language spaces - English, Italian, Russian and Chinese. To achieve this goal, a number of tasks were formulated, including:

1. to study the linguistic factor of the use of memes in the educational process,

2. to classify the ways of using memes depending on the purpose,

3. to analyze the content of memes in the liberal, ideological, as well as in the field of exact sciences.

The first part of the research made by Alexander Malakhov (PFUR) reveals the basic concepts associated with Internet memes. The second one written by Olga Matys (PFUR) is about using memes in a sphere of Russian liberal education in journalism. The third part of the research made by Ludovico De Serio (University of Genoa) presents an analysis using memes during studying math. The last but not the least part of this article made by Xie Dongqiang (PFUR) and reveals the aspects of an ideological studying using memes. This research was carried out using such scientific methods as analysis, comparison, description. In the course of the study, the authors used the work of such researchers as Susan Blackmore, G. Bini, M. Montagnani, Anna Zagoruyko, Maria Efremova, etc. Also there was used the content of sources such as VK publics "Memology: from antiquity to the present day" and "30 seconds before my expultion", GeoGebra, wnw.padlet.com, etc.
\end{abstract}

Key words: memes, education, journalism, mathematics, ideology, pop culture. 


\section{Introduction}

In the following article authors do a research in education and memes and their interactions. Covid-19 pandemic has greatly changed our usual way of life for many people, their home space has become an office, university and school. All this has contributed to the growth in the number of memes around the world, as new soil (Covid-19 pandemic) has emerged for their creation.

Meme is an information in one form or another (media object, i.e. an object created by electronic communication, phrase, concept), as a rule, witty and ironic, spontaneously gaining popularity, spreading on the Internet in various ways (through social networks, forums, blogs, instant messengers, etc.). Also denotes the phenomenon of spontaneous dissemination of such information or phrase. It came into use in the middle of the first decade of the 21 st century.

Memes can be both words and images. In other words, these are any statements, pictures, videos or sound sequences that have meaning, and is understood by people and are steadily distributed on the World Wide Web.

Not all information is subject to spontaneous uncontrolled spread from one Internet user to another, but only that which in some way leaves many users not indifferent to it.

Initially, anecdotes, jokes, links to content and files with media objects of a predominantly entertainment nature (first pictures, then flash clips, sound recordings, video recordings) were disseminated via the Internet in this way, but special attention was paid to the phenomenon later called "Internet memes". drew with the appearance of this name.

The Internet is such an important technology for memes that memes that are actively spreading through it have received a special name - Internet memes (jokes, anecdotes, etc., were successfully spread even before the invention of the Internet). Western meme researcher Susan Blackmore refers to memes for which modern communication technologies play a key role in the promotion of the term T-meme (Blackmore, 2008).

More often, internet memes are images with text, short videos, looping videos. Image memes could be two types. First one is image with text on the top and on the bottom of image. The second is image with text only on the top of the image. Image memes are also called image macros. Example of image macro - «One does not simply walk into Mordor» from Lord of the Rings. Short videos could be can be presented in the form of sketches with scripts, pranks and etc. Example of short video - Talking cat says Oh Long Johnson.

COVID-19 19 pandemic has greatly changed our usual way of life for many people, their home space has become an office, university and school. All this has contributed to the growth in the number of memes around the world, as new soil (the coronavirus pandemic) has emerged for their creation. Also, memes touched on the topic of education. Me and my Colleagues consider the media phenomenon of memes in the field of education and the educational possibilities of a meme as a way of simplified presentation of information. The integration of the education system, the Internet, and the media gave birth to new ways of information interaction between people. As the main element of images and visual culture in this era, memes are rapidly spreading in the network sphere, relying on the currently used media platforms (with Facebook 
and Instagram as the main ones, along with Zoom and Microsoft Teams and others), influencing people' every-day life and learning processes. There are also memes about the platforms themselves, among them it should be noted memes about platforms for organizing conferences such as Zoom, Skype, Microsoft Teams and etc. Also, memes can influence the effectiveness of student learning in an era of distance learning as the current one; therefore, the study of memes is of great practical importance in the educational process. We consider several ways of using memes in the educational process: a meme as a way to simplify educational material for better memorization, a meme as a way to learn a foreign language, and a meme as a student's reaction to the existing education system. Memes are a new type of carrier what can carry the content of student learning in visual representations; they can also be manipulated and used by teachers, and have been objects recognized and accepted by students since their inception.

It can be seen that as a medium of education for students, memes can effectively convey educational content and are an important bridge and link between teachers and students. Student educational activities are a hands-on and interactive process in which teachers and students participate together. It requires two-way communication and interaction between teachers and students. Memes are created in the process of communication and interaction between people. This growth process allows the meme to connect teachers and students and foster communication and interaction between them. With the help of memes, teachers can become closer and more understandable to students.

As the textual presentation of educational content is more formal and serious, teachers will inevitably appear constrained and alienated in the transfer of knowledge and learning. In the eyes of most people, the impression of teachers is relatively stereotyped. Seriousness, coldness are all words that describe teachers. Traditionally, teachers must win prestige in the hearts of students. However, as more and more young teachers emerge in education, young people are also the main users and disseminators of the meme. In this way, the use of the meme in education will shorten the distance between students and education can be more easily accomplished.

For example, many young teachers now use memes to awaken student enthusiasm for learning when evaluating homework and exam papers. Thus, grades in student work are no longer just numbers, but are vivid and interesting memes that reflect the teacher's full support and expectations for the students. Even if the child is criticized, it is no longer an attitude of disgust, but the courage to accept their mistakes, and then actively improve the learning attitude.

\section{Memes as a way of reflection of educational process}

The creation of original memes as a way of reflection on the educational process has become an integral part of liberal education in the field of journalism. This became especially noticeable during the Covid-19 coronavirus pandemic. Communicating most of 2020 on the World Wide Web during the regime of self-isolation and distance learning, many students, in particular, future journalists, intensified the process of creating memes on burning topics, responding to all changes in the educational process. Studying the content of two meme-publics of the popular in Russia social network VK "Memology: from antiquity to the present" 
(https://vk.com/memologists, 2015) of the Faculty of Journalism of Moscow State University and "30 seconds before my expulsion" (https://vk.com/30sdmo, 2018) of the Russian Peoples' Friendship University, the author of the study found that memes dedicated to the assessment of the learning process in universities, can be classified into several types, based on different grounds for classification.

The first basis for classification can be considered the language environment. Based on this, memes can be based on a foreign base (foreign films, TV series, and so on; a vivid example of such memes are Russified memes using the image of Shrek) and on a Russian-language base (Russian TV shows, bloggers, films, and so on). At the same time, memes play up exactly the learning process, using a meme template for the recognition effect. The next basis for classification is the actual use of the template. If a recognizable base is used for a meme, this meme can be considered a template. If the visual and verbal design of the meme is not associated with any popular image, and the comic effect is achieved due to a precisely noticed situation, such a meme can be considered original.

Authors of the research «Potential for using internet memes as a teaching tool» (Zagoruiko, Efremova, 2019) give much wider classification, noticing how the meme originated (deliberate, accidental, pop cultural), degree of versatility, structure (for example, characteristic memes, comparative memes), theme (education, politics, profession, etc.) and even method of exposure (comic, propagandistic, informational, etc.). If we use this classification for the current study, then memes in Russian language analyzed in this research can be classified as narrowly targeted for students and for students of journalism.

Here we should note that criticism of the learning process itself is a fairly common feature of memes about higher education. Students feel the need to express their dissatisfaction with any shortcomings in the organization of educational activities and create local memes on this topic. This trait of meme content has also escalated during the pandemic. If, before switching to remote mode, students complained, for example, about food at the university, an inconvenient lecture schedule and a long wait for an elevator, now they are expressing dissatisfaction with the technical equipment of the university and the lack of technical literacy not so much of the teaching staff as of the schedule dispatchers. At the moment, high workload has become the second most popular topic of memes - distance education turned out to be more tedious for many than usual, since the process of transition to a new education system was very fast, requiring further adjustments. Many teachers decided that the students had more time at home to work, and increased the number of home tasks and tests, which instantly caused a reaction from the students - to reflect on fatigue and discontent, they began to invent memes (Figures 1-4). 


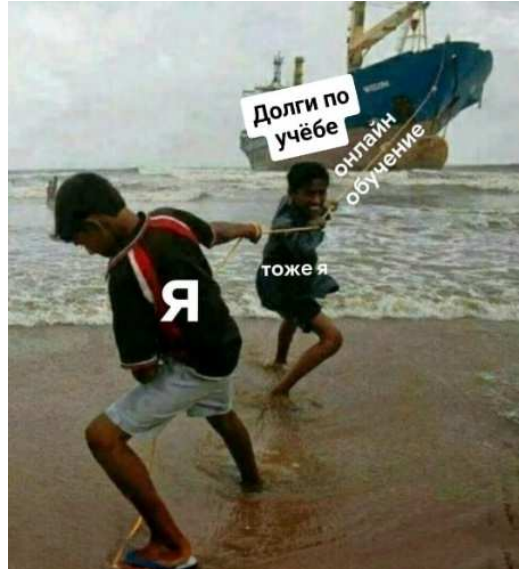

Figure 1 - "My academic debts - onlineeducation - me - also me"

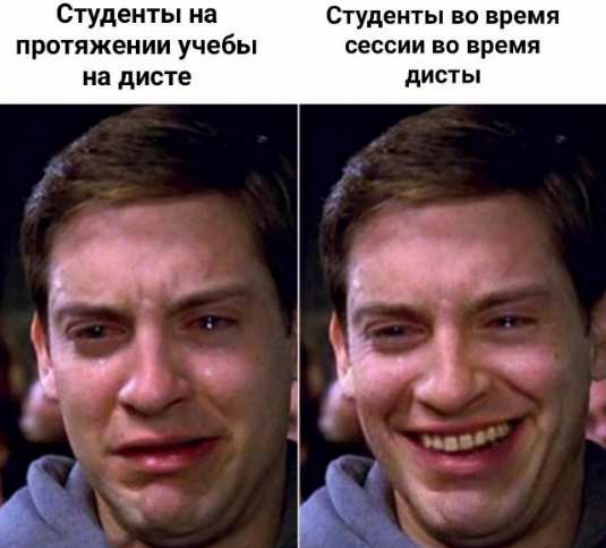

Figure 2 - "Students during distance education - Students during distance exams"

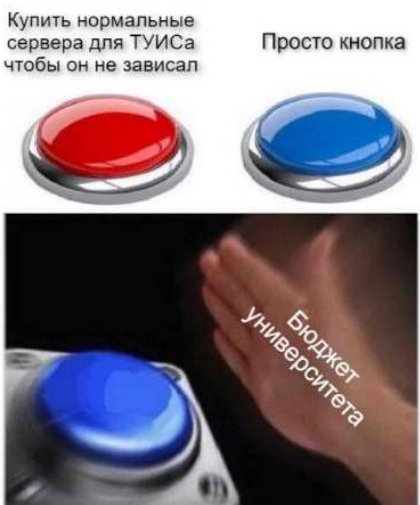

Figure 3 - "Button to buy fine server for university's site for testing could function just a button - University budget"

\section{Когда МГУ требует от преподов и студентов переход на новую непривычную и неудобную платформу}

Препод на следующей паре:

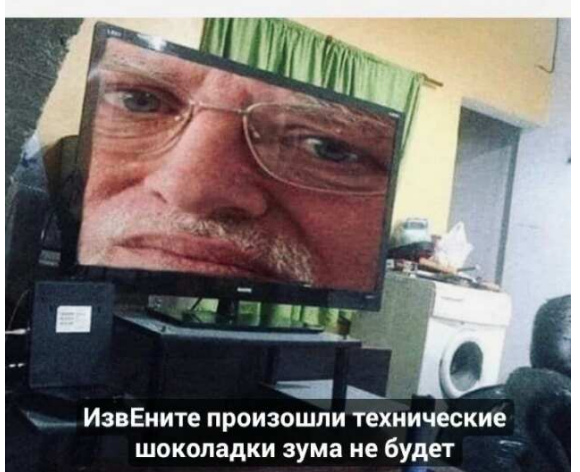

Figure 4 - "When MSU makes professors and students work from a new unusual and inconvenient platform - Professor next lesson: "eXcuSe me, there is a technical pUbLUm, there will be no zoom".

So, it is necessary to draw a number of conclusions about the creation of meme content used to reflect on the educational process. Firstly, memes on this topic allow students to release psychological stress during difficult learning, especially during a pandemic. Secondly, such memes can be studied by the organizers of the educational process to obtain the necessary criticism and change the organization system for the better. Thirdly, such memes serve as a source of alternative information about 
universities, which is not published on official websites, but which can influence the applicant's decision to apply to a particular university.

\section{Memes and mathematics, the Italian case}

As previously analyzed and asserted, memes are now a viral practice, capable of humor on all current topics, from politics to sports, to reality shows; and Italy is no exception in the acquisition of these modern communicative products.

Like every corner of the globe, Italy was also overwhelmed by the arrival and mainstream use of memes, initially relegated to certain areas of the internet (such as the 9gag and 4chan platforms) and limited in consumption due to the fact that they were mainly in English. Over the years, profiles began to emerge on the most popular social media (such as Facebook) in which memes were created for the Italian reader. Although at first, it was just a simple translation of memes produced in America from English to Italian, soon the contents began to turn towards themes and humor characteristic of the local national culture, starting to become a product loved not only by teenagers but appreciated by more and more people, although the younger generations remain the ones who best known and enjoy memes. This evolution has brought Italy " up-to-date" - if we want to say so - making memes part of popular culture as they are in almost all the world.

While maintaining the common goal of making people laugh, the topics on which users create memes have changed and evolved over the years: from simple funny images to tools for satire and expressing political opinions, to opportunities to show off specific knowledge in the many specialized groups that have sprung up spontaneously on the web. From this evolution in the use of this type of content, we then came to the point of thinking about how to use memes in education as an unconventional teaching tool, given the high coefficient of involvement and potential interest in young students, for whom they represent one of the most immediate communication tools.

In this regard, within a workshop dedicated precisely to alternative teaching practices, a group of students from the Department of Mathematics and Physics at the University of Turin presented research on the creation and classroom use of memes about mathematics, exploring the educational potential of memes as digital objects and expressions typical of contemporary Web 2.0 culture (Bini, et al., 2019).

This type of research becomes particularly interesting because it wants to try and demonstrate that in the twenty-first century even a scientific subject like mathematics is no longer the exclusive prerogative of traditional educational contexts, but can also be transmitted in a meaningful way through unconventional tools and not born for teaching practice, offering opportunities for connection between school mathematics and the language of students, giving value to youth culture.

Indeed, memes can offer students a less formal, but equally mathematically meaningful, environment to showcase their knowledge. In this environment, learners can demonstrate non-standard skills such as creativity, humor, and expertise in popular culture.

Integrating generationally and culturally distant objects into teaching practice certainly requires a degree of effort on the part of the teacher, since these virtual 
objects are well known and widely shared by young people who have internalized the logic, but can be difficult for people of other generations to handle.

Testing and possible uses in the classroom

The table 1 summarizes activities for students that include the use of math memes. The experiments carried out so far, which have involved middle school and high school students, have focused on the creation and discussion of memes to systematize previously acquired knowledge of students on a given curricular topic, with the educational goal of encouraging metacognition and the emergence of doubts or misconceptions. In the creation activity, students worked in pairs, creating their own memes with the Meme Generator site (imgflip.com), where it was possible to use networked devices, or working on paper cards prepared by teachers where online tools were not available. The phases of identification and explication of the specialized meaning were limited to a brief oral or written explanation, while the indepth phase involved a more in-depth development of the mathematical concept involved, also using other technological tools such as GeoGebra (a popular software for learning and teaching mathematics, geometry, algebra, and analysis), and was followed by a collective discussion led by the teacher (https://www.geogebra.org/).

\begin{tabular}{|c|c|c|c|}
\hline ACTIVITIES & \multicolumn{2}{|c|}{$\begin{array}{c}\text { IDENTIFICATION, } \\
\text { CLARIFICATION AND } \\
\text { DEEPENING OF MEANING } \\
\text { SPECIALIZED }\end{array}$} & $\begin{array}{c}\text { POSSIBLE } \\
\text { EDUCATIONAL } \\
\text { USES }\end{array}$ \\
\hline $\begin{array}{l}\text { 1. Searching the } \\
\text { web for a meme } \\
\text { on an assigned or } \\
\text { chosen topic }\end{array}$ & $\begin{array}{l}\text { Identification of } \\
\text { specialized } \\
\text { meaning }\end{array}$ & $\begin{array}{l}\text { Deepening of } \\
\text { specialized } \\
\text { meaning through } \\
\text { video, written }\end{array}$ & \multirow{3}{*}{$\begin{array}{l}\text {-Systematization of } \\
\text { knowledge } \\
\text { - Formative } \\
\text { assessment - } \\
\text { Metacognition }\end{array}$} \\
\hline $\begin{array}{l}\text { 2. Creating a } \\
\text { meme on an } \\
\text { assigned topic or } \\
\text { of your choice }\end{array}$ & $\begin{array}{l}\text { Explanation of } \\
\text { specialized } \\
\text { meaning }\end{array}$ & $\begin{array}{l}\text { presentation, } \\
\text { GeoGebra } \\
\text { applet }\end{array}$ & \\
\hline $\begin{array}{l}\text { 3. In-class } \\
\text { discussion of } \\
\text { memes found or } \\
\text { created by } \\
\text { classmates or the } \\
\text { teacher }\end{array}$ & $\begin{array}{l}\text { Identification } \\
\text { and explication } \\
\text { of specialized } \\
\text { meaning }\end{array}$ & $\begin{array}{l}\text { Deepening of } \\
\text { specialized } \\
\text { meaning through } \\
\text { collective } \\
\text { discussion }\end{array}$ & \\
\hline
\end{tabular}

Table 1, Bini \& Montagnini, 2019 


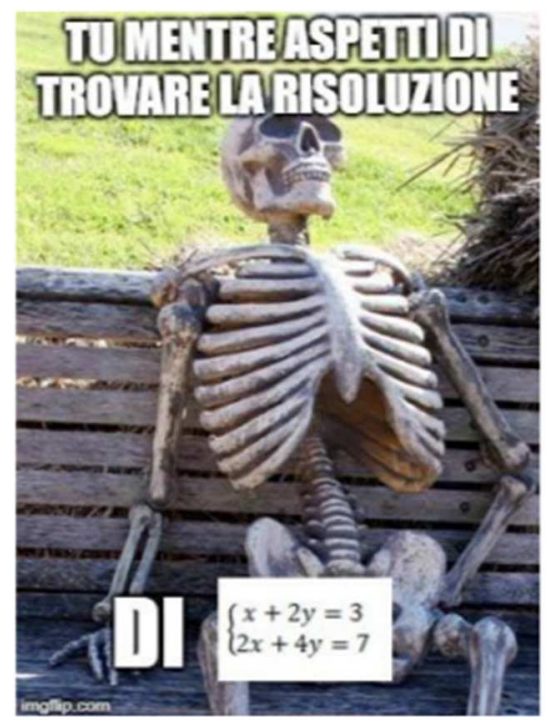

Figure 5 - An example of mathematical meme created by a student during the experiment [The inscription states "...you while you wait to find the resolution of...".].

The authors of the study also point out that in designing an activity with memes, it is good to keep in mind that these artifacts, although taken out of the social context and brought into the school environment, maintain their value as "social currency" within the class group and their message is reinforced by sharing and liking (Bini et al., 2019). It is therefore useful to set up space (online or offline) dedicated to sharing work that allows students to show their own creations, see those of others, and put likes. The most convenient online environment for sharing, which has been used in our experiments, is a Padlet board (padlet.com); if digital resources are scarce, memes can still be printed and hung in the classroom.

Activities involving memes in classrooms, as shown above, may have different approaches, but they are all linked by the fact that the protagonists are the students themselves. They are given the opportunity to unleash their creativity and explore a mathematical topic in different ways from those commonly adopted in the classroom, with the use of tools and languages are drawn from the social culture with which they come into daily contact outside the school walls. The teacher is invited to remain "behind the scenes" and take on the role of director and facilitator, allowing the transformation and adaptation of knowledge to new media.

\section{Memes in an ideological education, China case}

The meme, as a new thing created using technological advantages and the environment of time, has been loved by the public, especially young students, because of its many characteristics since its creation. With the constant expansion of technological advantages, a large number of memes of different categories and different nature appeared one after another. This on the one hand reflects the pace of social development, and on the other hand, it also reflects the frequency and love of the audience for the meme. Memes are increasingly becoming a "favorite" of the 
era of communication and communication between people and are gradually becoming an important part of people's daily lives. The production and use of memes have gradually made relevant meme research a hot topic in the academy's circle. To increase the attractiveness and credibility of student learning, it must be inseparable from fully understanding and using the "new favorites" of the time and applying them to education and learning. This is not only a direction that needs to be explored for the development of education itself, but also an inevitable attempt to match the psychology of students.

As a "nonverbal expression" of interpersonal communication in a postmodern network context, memes are a new type of medium and transmission of information, updated as interpersonal communication and communication develop. In terms of the development of information technology and the evolution of the external form of memes, memes have roughly passed through three stages of development and evolution:

1. Mostly symbolic expressions. :) Character expressions make full use of existing characters such as English letters, mathematical symbols, punctuation marks and Arabic numerals on computer keyboards and mobile phone keyboards, and combine them with expressions of facial emotions such as happiness, anger, sadness and other emotions, as well as with creative imagination, design and simulation, simulation of character expressions obtained by rearranging and combining elements of characters on the keyboard. After that, kaomoji appeared in Japan. Kaomoji also accepts a character expression as the main form of expression, mainly using a soft keyboard input method to create. facial expressions with character expressions spread in mobile phone text messages with their intuitive visual structure and playful, cute and vivid emotional expression, and become an intermediate element of the active atmosphere of people's communication. $\mathrm{O}\left(\cap_{-} \cap\right) \mathrm{O}, \wedge_{-} \wedge, \quad$ T_T (Zuo Hao. J).

2. Emoji. (-) : Emoji is represented by a yellow oval cartoon-like expression. It continues to imitate emojis of human facial expressions and more vividly imitates facial expressions or gestures when people experience different emotions. There is no need to spend time and effort on designing and creating symbolic expressions, just select and send directly, and the transfer of information will be faster and faster, which increases the efficiency of communication. Emojis gradually replaced kaomoji, represented by character expressions. different social networks and different input methods in different countries consistently set a set of small yellow facial expressions by default, indicating that emoji expressions have gradually become carriers for people to communicate information on various social networks.

3. Meme based on text and image. It mainly uses images as a key element, combines various communicative contexts, and is generated by secondary construction with specific words. the main elements, from the initial direct selection to self-creation by users of the network, are mostly borrowed from popular online languages, screenshots from movies and TV works, celebrities, hot events, etc. With the expansion of the benefits of Internet technology, the creation, use, and dissemination of memes has gradually evolved into a powerful popular culture. Currently, the frequency of meme using and the constant expansion of the audience has led to the fact that memes have expanded and turned into emoticons in a broad sense.

As an intermediary in interpersonal communication, memes can convey information about the interacting party. In the context of interpersonal online communication, 
memes have gradually become a widely used medium of information. A meme can construct and implicitly change the meaning of transmitted words, and can also compensate for communication barriers caused by simple text transmission. Vivid images make it easier for both parties to reach a consensus on the persuasiveness of a semantic space that meets the needs of in-depth communication of students ' educational content. As a new type of medium, memes can carry the content of students ' learning in visual representations; they can also be manipulated and used by teachers, and they have been objects recognized and accepted by students since their creation. It can be seen that as a carrier of ideological and political education for students, memes can effectively convey educational content and are an important bridge and link between teachers and students.

Students' educational activities are a hands-on and interactive process in which teachers and students participate together. it requires two-way communication and interaction between teachers and students. Memes are created in the process of communication and interaction between people. This growth process allows the meme to connect teachers and students and promote communication and interaction between them. This connection function of the meme is mainly manifested in the ability to connect the emotions of both.

Teaching students is not only a process of systematic exchange of knowledge with students, but also the process of educating them to be faithful to the good and beauty. This process must necessarily be accompanied by rich emotions that allow you to realize the favorable interaction between teachers and students. Meme is a new educational tool that provides more extensive materials for communication between teachers and students. Since the textual representation of the content of education is more formal and serious, teachers will inevitably appear constrained and alienated in the process of knowledge transfer and learning. A meme gives people a visual impact through the arrangement and combination of its images or text, and its concise content and intuitive representation of emotions make the meaning of substitution and existence in the context of communication more sufficient. Through visual representation, students ' visual perception organs are mobilized to create a strong emotional vibration. as for specific educational content, the meme uses its own display of symbolic meaning and emotional substitution, thereby creating opportunities for aspiring teachers and students to participate in student learning.

Thus, the display and transmission of educational content using emoticons is more emotional and vivid than simple text expressions. This can stimulate the enthusiasm and initiative of college students to participate in educational exchanges, and can also reduce the alienation of personality between educators and students to enhance the emotions between the two parties, thereby effectively connecting them.

The process of ideological and political education of students is the process of improving the ideological and political quality of students. And students, as one subject with a strong initiative in the process of ideological and political education of students, have a call to discourse to maintain their own discursive power. In the process of obtaining educational information, they seek to free themselves from the power of discourse, hoping to realize an equal dialogue with teachers.

Memes can be used in the field of ideological and political education of students because of the good communication stories and situations created by their unique visual image function. On the one hand, memes are very emoticon-mimicking, using 
a variety of interesting design styles to achieve a beautiful representation of content and emotions. As a result, the meme has a vivid plot and context, and communication and use of the meme is just an interaction between different emotions, views and values. The use of this method of expression compensates for the authority and seriousness of the educational content presented only in words, and can increase the attractiveness of education. This can activate and mobilize the enthusiasm and initiative of students to participate in the educational process. Innovative expression of educational content will inevitably attract the attention of students. In the process of receiving emoticons of ideological and political education, they must interpret memes that carry educational content in order to ensure cooperation and dialogue with educators in an educational atmosphere. On the other hand, memes give image elements or text elements a new meaning through a variety of discourse constructs. As the volume of communication and distribution gradually expands, some memes also have different semantic spaces. The meaning of such a discourse is hidden in the expression, and it can protect the real thoughts and emotions of students. Memes use this emotional ambiguity to freely express their thoughts, emotions, and opinions to enable viewing and sharing of educational content.

The development of education is not a static process. its own development and practicality are important factors that contribute to the continuous creation and renewal of education. the ideological and political education of students, as an educational practice for solving doubts and puzzles for students, will inevitably develop continuously with the progress of time, which is determined by its own nature. With the acceleration of time, the development of mobile Internet technologies and the advent of big data, people's lives, studies and work have undergone huge changes. Under the conditions of this era, the methods of teaching students should also be changed accordingly. all this indicates that the use of memes in the educational process of students allows you to create a good communication atmosphere and implement the requirements of modern development of educational media.

Memes are a product of the needs of interpersonal social networks on the Internet in the context of the development of mobile Internet technologies and are a language tool for the general public, especially for students, to reach consensus on social networks on the Internet. It implements the expansion of human modal expressions in virtual interpersonal communication and makes up for the absence of the human body. Self-carrying function and communication function interpersonal function of creating identity and function of visual modifications make memorial weather vane, reflecting the culture, thoughts, emotions, opinions and other content in a certain way. Students, as the main users and distributors of memes, represent a key group that promotes memes among the population. Ideological and political education of students fully monitors the behavior of students, closely monitors their ideological tendencies and uses methods that interest students to conduct educational practice. Only in this way can firmly capture students and direct them to actively and consciously participate in educational activities. 


\section{Conclusion}

In this article the group of authors made a list of important conclusions about the phenomena of using Internet-memes in a sphere of education. First of all, they consider memes as an effective tool to catch students' attention during lessons. Especially it can work for studying foreign languages and strengthening connection between teacher and students. Then, memes about education in universities can be a source of inside information and source of reflection about education process, that gives an opportunity to relax. In a sphere of math educational memes are new and unusual way to unleash the creativity of students and explain them some difficult moments. Then memes are considered as a potential and progressive useful tool for ideological education.

\section{References}

Bini G., Montagnani M. (2019) Comprendere, creare e utilizzare in classe i meme matematici, Torino.

Blackmore, S. (2008) The trouble with Memetics. Skeptical Inquirer, 32,65

Zagoruiko A. O., Efremova M. A. J. (2019). The potential of using Internet memes as a learning tool. Questions of teaching methods in higher education, 8 (28), 12-21.

Zuo Hao. J. (2008). The characteristics of memes and their influence on students' ideological and political education. Journal Longyuan, No. 08, 114-115.

\section{Web references}

Blackmore, Susan, (2008), Memes and Temes. [Online] Available: https://www.ted.com/talks/susan_blackmore_on_memes_and_temes/transcript?language =en\#t-1845

GeoGebra. [Online] Available: https://www.geogebra.org/ (visited at 16.12.2020)

Meme generator website IMGFLIP. [Online] Available: imgflip.com (visited at 16.12.2020)

Online platform for teaching PADLET. [Online] Available: padlet.com (visited at 16.12.2020)

VK-public "Memologia: ot antichnosti do nashikh dnej». [Online] Available:

https://vk.com/memologists (2015)

VK-public "30 secund do moego onchisleniya». [Online] Available:

https://vk.com/30sdmo (2018) 